\title{
Local Deformation and Failure Mechanisms of Polymer Bonded Energetic Materials Subjected to High Strain Rate Loading
}

\author{
Suraj Ravindran $^{1} \cdot$ Addis Tessema $^{1} \cdot$ Addis Kidane $^{1}$ (B)
}

Received: 30 October 2015/Accepted: 18 January 2016/Published online: 29 January 2016

(C) Society for Experimental Mechanics, Inc 2016

\begin{abstract}
The dynamic multi-scale deformation mechanism of polymer bonded energetic material is investigated. Samples made of polymer bonded sugar (a known simulate for polymer bonded explosives) with $85 \%$ sugar crystals and $15 \%$ polymer binder are used. The samples are dynamically compressed using a split Hopkinson pressure bar. Using a high magnification, meso-scale 2D digital image correlation experimental setup, the local deformation is measured in situ at sub-grain scale. The macroscale deformation mechanism is also investigated with the help of 3D digital image correlation. From the mesoscale experiment, it is observed that the local strain distribution in the specimen is highly heterogeneous with large strain localization occurring at the polymer rich areas between the crystal boundaries. Deformations of the majority of the crystals are minimal, and usually realign themselves to accommodate large deformation of the binder by rigid rotation and sliding. Due to this, delamination of the polymer binder from crystals and binder cracking are the main local failure modes. It is also observed that the presence of small crushed crystals from material processing are the favorable sites for this opening mode failure.
\end{abstract}

Keywords Energetic materials - Dynamic experiments . Mesoscale · PBX · PBS

Addis Kidane

kidanea@cec.sc.edu

1 Department of Mechanical Engineering, University of South Carolina, 300 Main Street, Columbia, SC 29208, USA

\section{Introduction}

Dynamic characterization is critical in granular materials, such as polymer bonded explosive (PBX), concrete, sand, armor protection materials etc., employed in extreme loading conditions. Especially in the case of polymer bonded explosives, understanding the failure mechanism under high strain rate loading is vital for safe use of these materials. PBXs typically contain $60-95 \%$ of explosive solid loading and about 5-40 \% of the soft polymer binder. Due to significant mismatch between the mechanical properties of the solid constituents and the soft polymer binder, the presence of inclusions, cracks, and voids from the processing of the material, the failure mechanism of PBXs is complex and extended to multi-length scales. Quantifying the local deformation and failure initiation mechanisms of PBXs subjected to dynamic loading at meso-scale entails a deep understanding of the material response at much smaller length scales than macroscale measurements.

Optical methods, such as photoelasticity, digital speckle radiography, laser induced fluorescent speckle photography, and digital image correlation have been used in the past to understand the failure and fracture mechanics of PBXs subjected to dynamic loading [1-7]. Most of these studies are at macroscale and have provided much insight into the continuum scale high strain rate behavior of PBXs [2-10]. However, these experiments are inadequate to understand the local deformation mechanisms in highly heterogeneous PBX materials. On the other hand, high spatial resolution micro scale experiments have been proven to elucidate the local deformation mechanisms in these materials under quasi-static loading conditions [11, 12]. Although high spatial resolution full field DIC measurements based on optical microscopes or scanning electron 
microscopes have been successfully implemented to measure local deformation in different materials at submicron scale under quasi-static loading conditions [12-17], high temporal resolution measurement with high spatial resolution are not established. Due to the absence of a suitable experimental method, the local behavior of these materials under dynamic loading conditions is not well understood although it is critically important. Recently an optical based meso-scale digital image correlation has been proposed that can be used to measure the local deformation in heterogeneous materials $[18,19]$.

In this study, an experimental setup based on mesoscale digital image correlation is used and the local deformation and failure mechanisms in PBXs at high strain rate loading are investigated by measuring the local strain fields at subgrain scale level. To the best knowledge of the authors, this is the first detailed work on understanding the local deformation of PBX by measuring strain at the sub-grain scale and at high temporal resolution. Macroscale experiments are also conducted and the failure mechanisms at macro and meso scales are compared.

\section{Materials and Methods}

\section{Material Preparation}

The material used in this study is polymer bonded sugar (PBS) crystal, a known simulant of polymer bonded explosives. Sugar is far from representing the actual microstructure of RDX and HMX, but is still an ideal and widely used simulant for the following reasons, (1) macroscopically the deformation behavior of polymer bonded sugar is similar to most polymer bonded explosives [20] and, (2) microscopically the monoclinic structure of sugar resembles the monoclinic crystalline structure of high melting point explosives (HMX) [21], though the deformation mechanism have not been confirmed to be identical. In addition, its simplicity and safety make sugar a convenient surrogate for explosive materials that can be tested in university laboratories and facilities. On the other hand sugar is soluble in water therefore moisture and humidity can affect its mechanical and physical properties. In our work, proper care was taken to minimize the effect of moisture and humidity during fabrication and characterization process. Samples were cured in a vacuum assisted oven. Specimens were dry polished without the application of water. Experiments were conducted in a controlled atmosphere of temperature and humidity.

The formulation is composed of 85/10.8/2.89/1.31 wt \% of sugar/Hydroxyl-terminated polybutadiene (HTPB)/dioctyl sebacate (DOS)/Toluene diisocyanate (TDI). HTPB is the monomer, DOS is the plasticizer and TDI is a curing agent. HTPB is a common binder material in polymer bonded explosives. To study the effect of loading rate on the failure behavior of PBX one should account for the strain rate sensitivity of the typical viscoelastic polymer used in PBX [22]. The intention of the current work however, is not to study the effect of strain rate rather study the local deformation mechanism of PBX at a given loading rate by measuring the deformation in and across the boundary of the crystals. It is a first of its kind, and intentionally other parameters, such as strain rate are excluded.

Fabrication of the PBS specimens involves three major steps. First, the HTPB is mixed with the plasticizer (DOS) and curing agent (TDI). Then the sugar crystals are added to the blend and mechanically mixed thoroughly. It is then kept in a vacuum oven for $24 \mathrm{~h}$ at $60{ }^{\circ} \mathrm{C}$, where the partial curing leads to the formation of a powdery mixture. Using a cylindrical mold of bore diameter $25 \mathrm{~mm}$, the powder is then hydrostatically pressed at $90 \mathrm{MPa}$ to cylindrical billets. These billets are completely cured by keeping them in the oven for $120 \mathrm{~h}$ and cooled naturally to room temperature. The specimens for the experiments are then machined from billets using a milling machine.

\section{Experiment Setup}

\section{High Strain Rate Testing}

The high strain rate compression experiments are performed using split Hopkinson pressure bar (SHPB) apparatus. Since the specimen used in the experiment is made of low impedance material, a soft polymer binder, polycarbonate bars are used as input and output bars to minimize the impedance mismatch and obtain appreciable transmitted signals [23]. Both the incident and the transmitter bars are made of $1830 \mathrm{~mm}$ long and $25.4 \mathrm{~mm}$ diameter polycarbonate bar. To reduce the friction between the specimen and the bars, a thin layer of molybdenum disulfide is applied on the contacting surface. The waves in the incident and transmitter bars are measured with the help of strain gages located at the middle of the incident and transmitter bars. The average strain rate, strain and stress on the specimen are obtained using well-known equations,

$\dot{\varepsilon}_{s}(t)=-\frac{2 C_{b}}{l_{s}} \varepsilon_{R}(t)$

$\varepsilon_{s}(t)=\int_{o}^{t}-\frac{2 C_{b}}{l_{s}} \varepsilon_{R}(t) d t$ 
$\sigma_{s}(t)=\frac{E_{b} A_{b}}{A_{s}} \varepsilon_{T}(t)$

where $l_{s}$ is the original length of the specimen, $\varepsilon_{R}(t)$ is the time resolved strain of the reflected pulse in the incident bar and $C_{b}$ is the velocity of the wave in the bar. $E_{b}$ is the elasticity modulus of the bar material, $A_{b}$ is the area of cross section of bar, $A_{s}$ is the area of cross section of specimen. It should be noted that the stress-strain plots are used to see the trend, and are not critical for this study. Wave attenuation and dispersion in the bars are not corrected in the analysis.

\section{High Speed Imaging}

To observe the local deformation in situ, a high speed camera equipped with high magnification extension tube is used. Figure 1a shows the schematic representation and Fig. 1b, shows the close up picture of the actual setup used in the experiment. A high speed camera SAX2, by Photron Inc., at a framing rate of 100,000 frames/s and at a resolution of $384 \times 264$ pixels is used. For high magnification imaging, an extension tube from Navitar is used. The greatest challenges in obtaining images at high magnification and high rates are low depth of field of the optical system and the need of high intensity illumination. For illumination, two high intensity LED lights and a fiber optic light tip were kept as close as possible to the specimen without obstructing the view of the camera as shown Fig. 2b. This illumination provides sufficient light to acquire the images, up to 180,000 frames per/second. An optical resolution of $10 \mu \mathrm{m} /$ pixel is achieved using the proposed method, which is sufficient to obtain strain field inside a grain of size $400 \mu \mathrm{m}$ using DIC. The macroscale deformation of the specimen is measured using stereovision system, comprise of two SAX2 high speed camera and two $100 \mathrm{~mm}$ lenses. In this case, two LED cold lights are used for illumination. The image acquisition rate is kept same as that of the mesoscale dynamic experiments. Details of the optical parameters used in the two systems can be found in Table 1 .

\section{Specimen Geometry and Surface Preparation for Macro and Meso Scale DIC}

To facilitate DIC measurement, a high contrast, random and isotropic speckle pattern has to be applied on the surface of the specimen. It is very important to choose the right size speckles according to the image resolution of the optical system and experimental setup. As a thumb of rule' for good displacement resolution and accuracy, every
Fig. 1 a Schematic of experiment setup, b Close up image of the experiment setup (a)
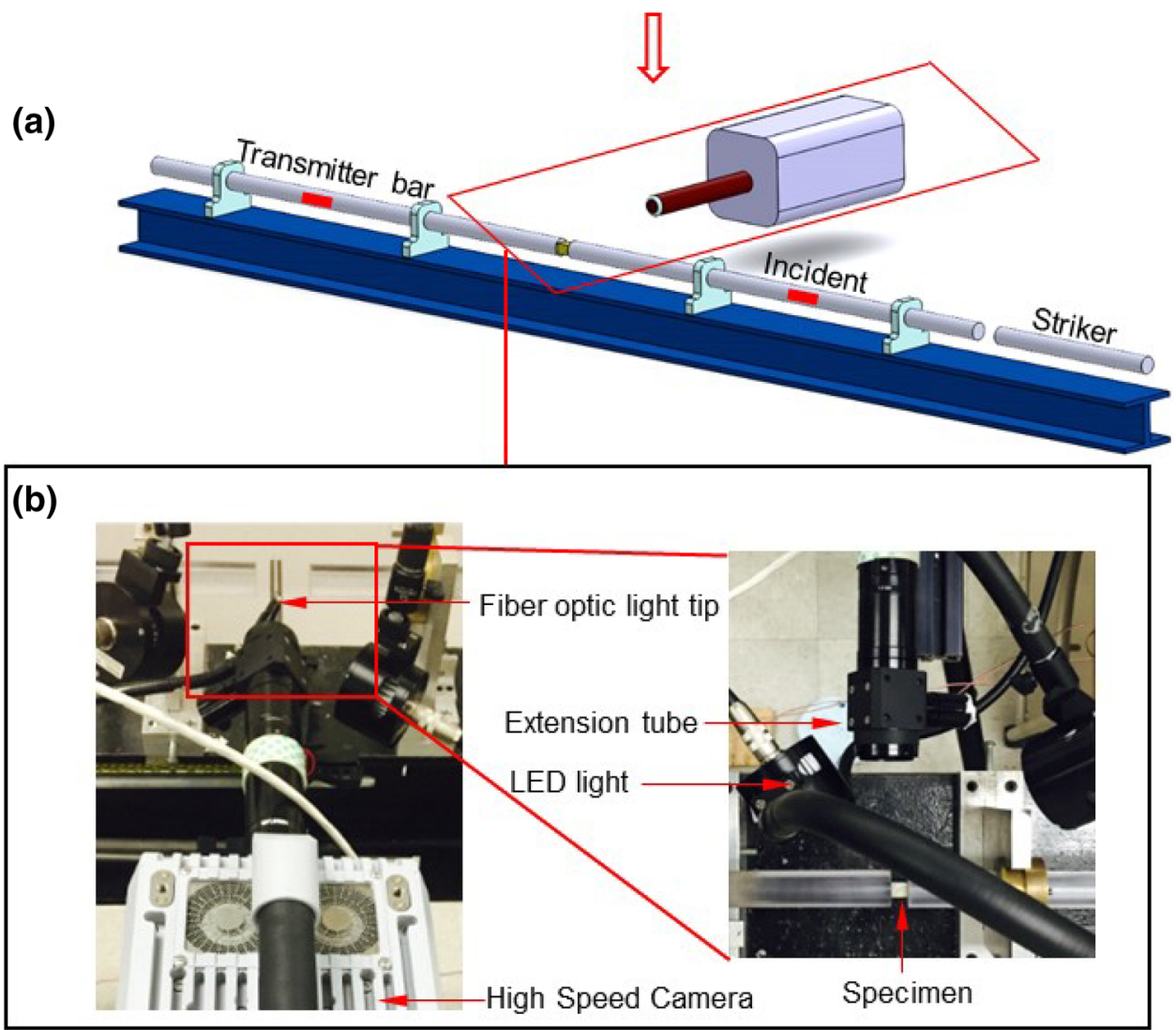

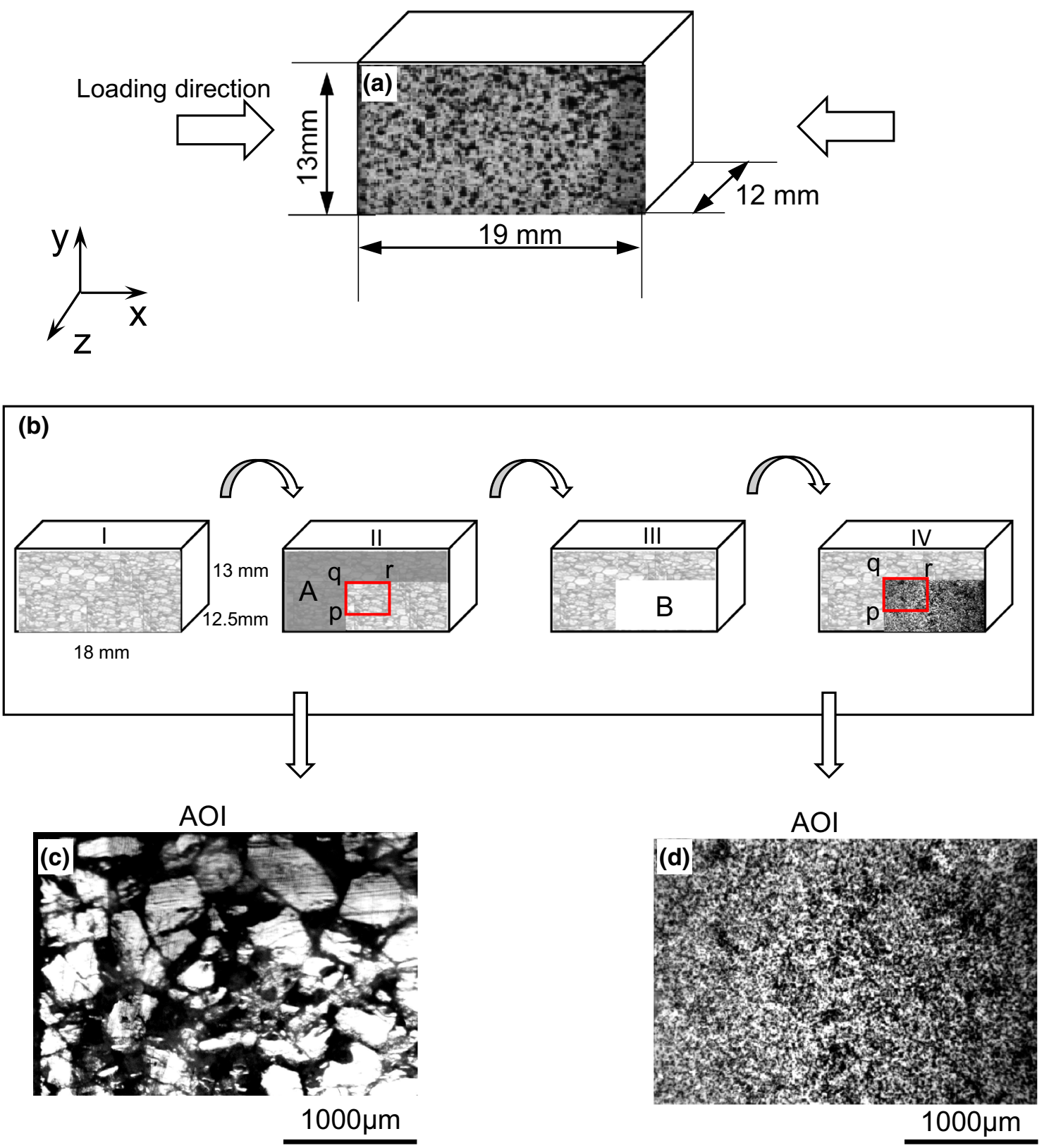

Fig. 2 a Specimen dimension and speckle pattern for macroscale experiment, b Specimen dimension and steps involved in marking and speckling the area of interest (AOI) for mesoscale experiment, $\mathbf{c}$ Microstructure of AOI, $\mathbf{d}$ Speckle pattern of AOI

Table 1 Image framing rate, temporal resolution, and field of view of the macro and mesoscale DIC setup

\begin{tabular}{lll}
\hline Experiment details & Macro & Meso \\
\hline Image acquisition rate (frames/second) & 100,000 & 100,000 \\
Image resolution $($ pixels $\times$ pixels) & $384 \times 256$ & $384 \times 256$ \\
Pixel to length ratio $(\mu \mathrm{m} /$ pixel) & 150 & 10 \\
Field of view $(\mathrm{mm} \times \mathrm{mm})$ & $30.72 \times 21.12$ & $3.84 \times 2.64$ \\
DIC algorithm & $3 \mathrm{D}$ & $2 \mathrm{D}$ \\
\hline
\end{tabular}

speckle has to be sampled by at least 3-5 pixels [24]. The image resolution for the macroscale experiment is $150 \mu \mathrm{m} /$ pixel and, therefore, a speckle size of $500-800 \mu \mathrm{m}$ is required. These speckles are achieved using an airbrush and flat paint. First a thin layer of white paint is applied on the surface of the specimen, and after it has dried 
completely, a black paint was sprayed on the top of the white layer using an airbrush. The specimen dimensions and the speckle pattern for the macroscale DIC measurement are shown in Fig. 2a.

The mesoscale digital image correlation provides the capability to capture strain fields at grain scale level. It is very important to mark the area of interest (AOI) precisely to relate strain evolution with the underlying microstructure. For that matter, three major steps, polishing, marking, and speckling is followed as illustrated in Fig. 2b. First, the specimen was dry polished with silicon carbide paper of grit size varying from 240 to 1200 and then finely polished with diamond particles of $3 \mu \mathrm{m}$. Secondly, in order to mark the AOI and obtain a microstructural image, the left and top part of the specimen is covered with scotch tape as $\mathrm{L}$ shape (A), as shown in Fig. 2b (II), leaving the microstructure of the right bottom side of the specimen accessible for imaging and speckling. Note that the right edge of the vertical scotch tape (pq) and the bottom edge of the horizontal scotch tape (qr) are used as a reference, during imaging both the microstructure and DIC images. The image of the microstructure is captured covering entire the field of view of the area of interest marked with red rectangle shown in Fig. 2b (II). The microstructure of the AOI is shown in Fig. 2c. In step three, the AOI is speckled for DIC measurements. In order to speckle the specimen, first a thin layer of white paint was applied, using an airbrush, on the specimen surface covering the AOI, and allowed to dry partially. Then a black toner powder is deposited on top of the partially dried white paint, by blowing the powder onto the surface using an air gun, and allowed to dry completely. Finally, the scotch tape was peeled off, which gave speckle patterns of AOI shown in Fig. 2b(IV), d.

Using these speckled specimens both the macro and meso scale experiments are conducted, while images are taken in situ. The camera system was triggered with the help of oscilloscope and strain gages attached on the input bar. Using the camera delay time feature, the duration of the trigger is adjusted to capture images prior (reference image) and after deformation. The reference and the consequence images are then imported for further post processing following a well-documented procedure available in the literature [24-26].

\section{Post Processing of Macro and Meso Scale DIC}

For macroscale strain field measurement, the images are processed using Vic3D, commercial digital image correlation software by correlated solutions Inc. As mentioned above the resolution of the macroscale experiment is $150 \mu \mathrm{m} /$ pixel and the average speckle size is $600 \mu \mathrm{m}$. Hence, subset sizes of $1.95 \times 1.95 \mathrm{~mm}^{2}$ are used, considering a minimum of $3 \times 3$ speckles in one subset. Strain calculation in this study is done with the exhaustive search mode, which enables the highest amount of data recovery when correlation fails, at the expense of processing time [24]. A higher order interpolation function (Optimized 8-tap) is used to convert discrete digital data points to continuous data. The correlation criterion was chosen to be zero normalized, which is insensitive to the scaling of light intensity.

Similarly, in the case of mesoscale strain field calculation a commercial 2D digital image correlation software, Vic 2D, is used. The strain calculation, in this case, was done with the exhaustive search mode similar to the macroscale. However, a subset size of $110 \times 110 \mu \mathrm{m}^{2}$ is employed in this case which enables to have at least $\sim 14$ subsets in one grain of $400 \mu \mathrm{m}$ size.

The proposed surface measurement cannot provide detailed failure mechanisms that occur below the considered plane. Especially, it is difficult to tell where the failure initiated, an interesting subjected but out of the scope of the current work. On the other hand, the strain localization and the deformation profiles in and across the boundary of the crystals are investigated, by locally measuring the deformation on the binder and crystals in the same plane. The images are captured on a plane perpendicular to the loading direction. Considering the loading and boundary conditions in a Hopkinson bar experiment, it is reasonable to assume that, macroscopically, the deformation is fairly uniform across the thickness of the specimen. This is the basic assumption of Hopkinson Pressure bar experiment. The proposed local measurements will show that the deformation is heterogeneous locally, whilst being fairly uniform macroscopically. In addition, the surface measurements, in conjunction with the underline microstructure, can give information about the interaction and propagation of strains between the binder and crystals, which is the main objective of the current work.

It was also found that the effect of subsurface deformation on the measured surface deformation is negligible, in the early stages of the loading. The specimens are unconfined and free to expand in the out of plane direction. If there is out of plane motion due to subsurface deformation, the captured images would have been out of focus, as the depth of field of the microscope is very small. Fortunately, we haven't observed such a large effect during the early stage of the deformation and all the images correlate well. However, once failure initiated, the images are no longer in focus and could not be used for digital image correlation. So, it is reasonable to assume that the early stage of deformation is one dimensional and the deformation fields presented in this work can representative the deformation profile across the thickness of the specimen. 


\section{Results and Discussion}

\section{Macro Scale Deformation Behavior}

Typical stress-strain relation of PBS subjected to dynamic loading is shown in Fig. 3a. The strains calculated based on the strain gage readings and obtained from 3D DIC are compared in Fig. 3b. The values are comparably close to each other especially in the early stage of deformation; however, there is a small variation in the later deformation stage of the material, which could be due to the variation of the strain field due to speckle cracking once the failure initiation occurs in the material. To check the stress equilibrium on the flat specimen tested at the macroscopic scale, three wave and single wave analysis is performed. As shown in Fig. 3a, stress obtained from the three wave analysis oscillates around the stress obtained from the one wave analysis indicating reasonable stress equilibrium in the specimen.

Figure 4 shows the contour plot of maximum shear strain fields as a function of time obtained from macroscale DIC. It is clearly visible that at $200 \mu \mathrm{s}$, high shear strain localization is started at each corner of the specimen and later merged into the center of the specimen leading to shear band failure.

The shear band formation is clearly visible in the fracture specimen shown in Fig. 4c. Apart from observing the macroscale shear band formation, it was impossible to look into the local deformation mechanism at this scale. The onset of local deformation mechanism is observed in the mesoscale experiment and discussed in the next section.

\section{Mesoscale Deformation Behavior}

Highly heterogonous deformation is apparent in the PBS sample tested, and the experimental method employed was able to capture these local strain fields accurately. Figure 5 shows typical local axial, lateral and shear strain fields in the PBS specimen subjected to dynamic compressive loading. It is distinctly visible that, the local compressive strain in some regions reach as high as $8 \%$ for the global axial strain of only about $0.5 \%$. Similar observations have been made in the meso scale computational study of polytetrafluoroethylene-Al-W granular composites under dynamic loading conditions [27]. The strain localization is prevailing around crystal boundaries, rich in polymer, and it is presumable that most of the deformation was accommodated by the deformation of the soft polymer binder while crystals realign themselves to accommodate the deformation. This could be the reason that the stress-strain plot has extended deformation after the yield. Furthermore, from the lateral strain field shown in Fig. 5b, it is clearly seen that the local deformation occurs at an angle close to $\pm 45^{\circ}$, indicating local shear band formation is also captured in the experiment.

In order to understand these effects better, the local strain fields were compared with the underline microstructure taken before the deformation as shown Fig. 6. It is shown that, in most cases, the axial strain localization occurs at the vertical or low angle interfaces with thick binder interface, where there are no crystalcrystal contacts or adjust crystals are far away from each other, for example between Crystal 3 (C3) and Crystal 11 (C11), between Crystal 4 (C4) and Crystal 9 (C9), between Crystal 7 (C7) and Crystal 5 (C5), between Crystal 8 (C8) and Crystal 6 (C6), between Crystal 10 (C10) and Crystal 14 (C14) etc. It can be seen that crystals C5, C6, C9, C11, $\mathrm{C} 12$ and $\mathrm{C} 13 \mathrm{C} 14 \mathrm{C} 15$ are in contact and the strain localization observed at the interface between these crystals are minimal due to the absence of polymer binder. It is visible that the axial strain field in these crystal interfaces are small and reasonably continuous compared to the
Fig. 3 a Stress strain curve for $\mathrm{PBX}$ at $300 \mathrm{~S}^{-1}$. b Comparison of DIC strain measurement with the strain gage measurement, figure shows the strain versus time
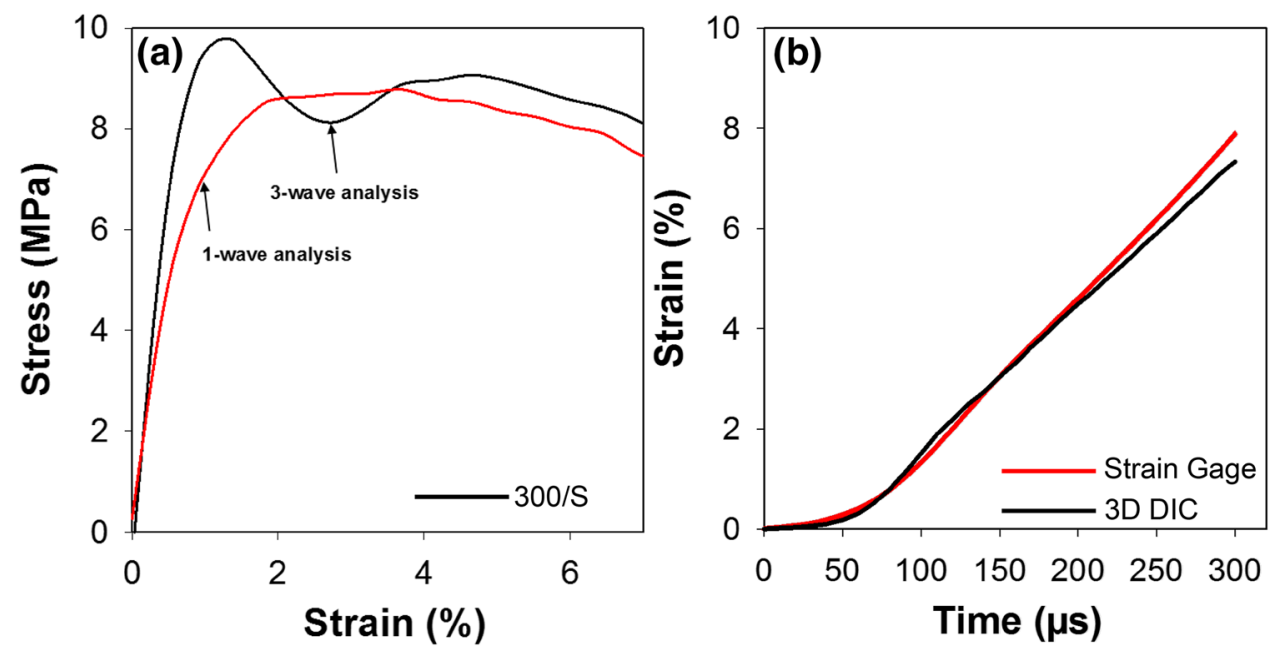


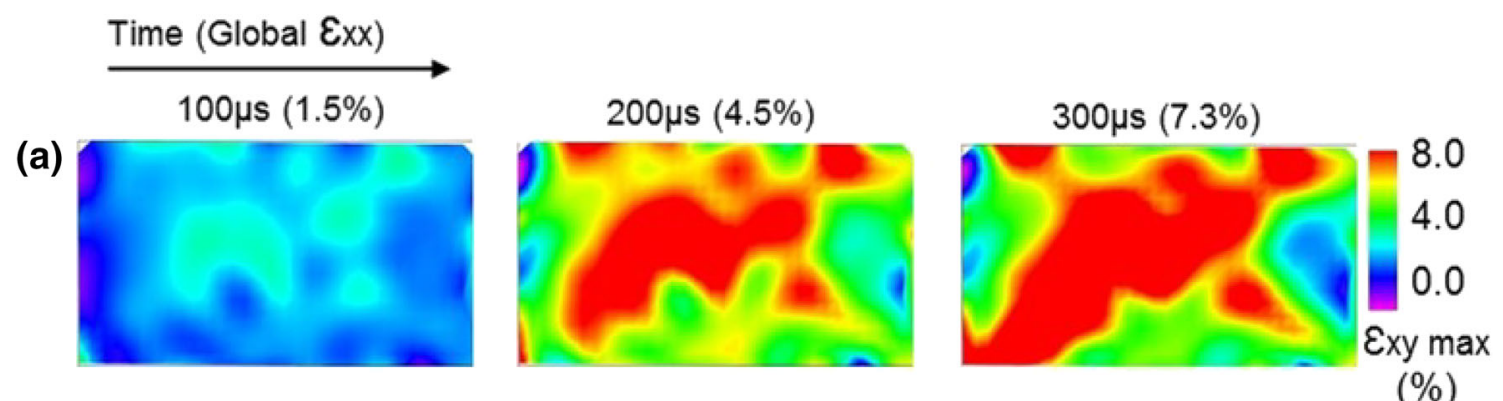

(b)

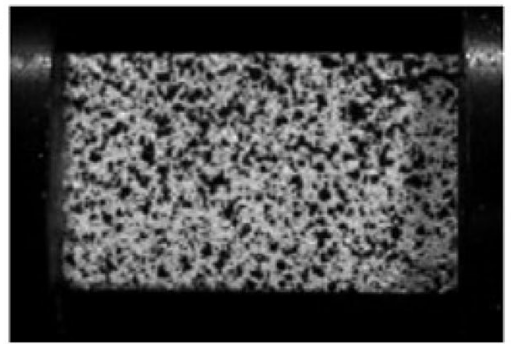

(c)

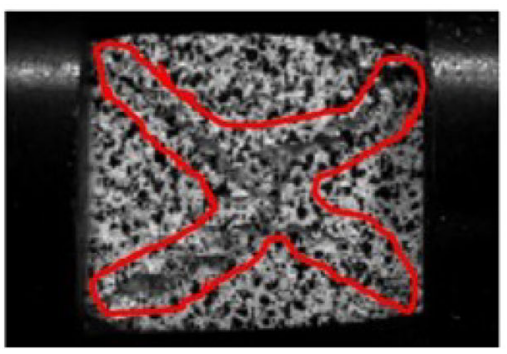

Fig. 4 a Maximum shear stress in the material at 100, 200, $300 \mu$ s from the time of loading and specimen $\mathbf{b}$ in undeformed condition and $\mathbf{c}$ at failure state

Fig. 5 Local strain fields a axial, b lateral and, c shear at different time, $60,120,160 \mu \mathrm{s}$ after loading with corresponding global axial strains, $0.5,1.8,3.5 \%$ respectively

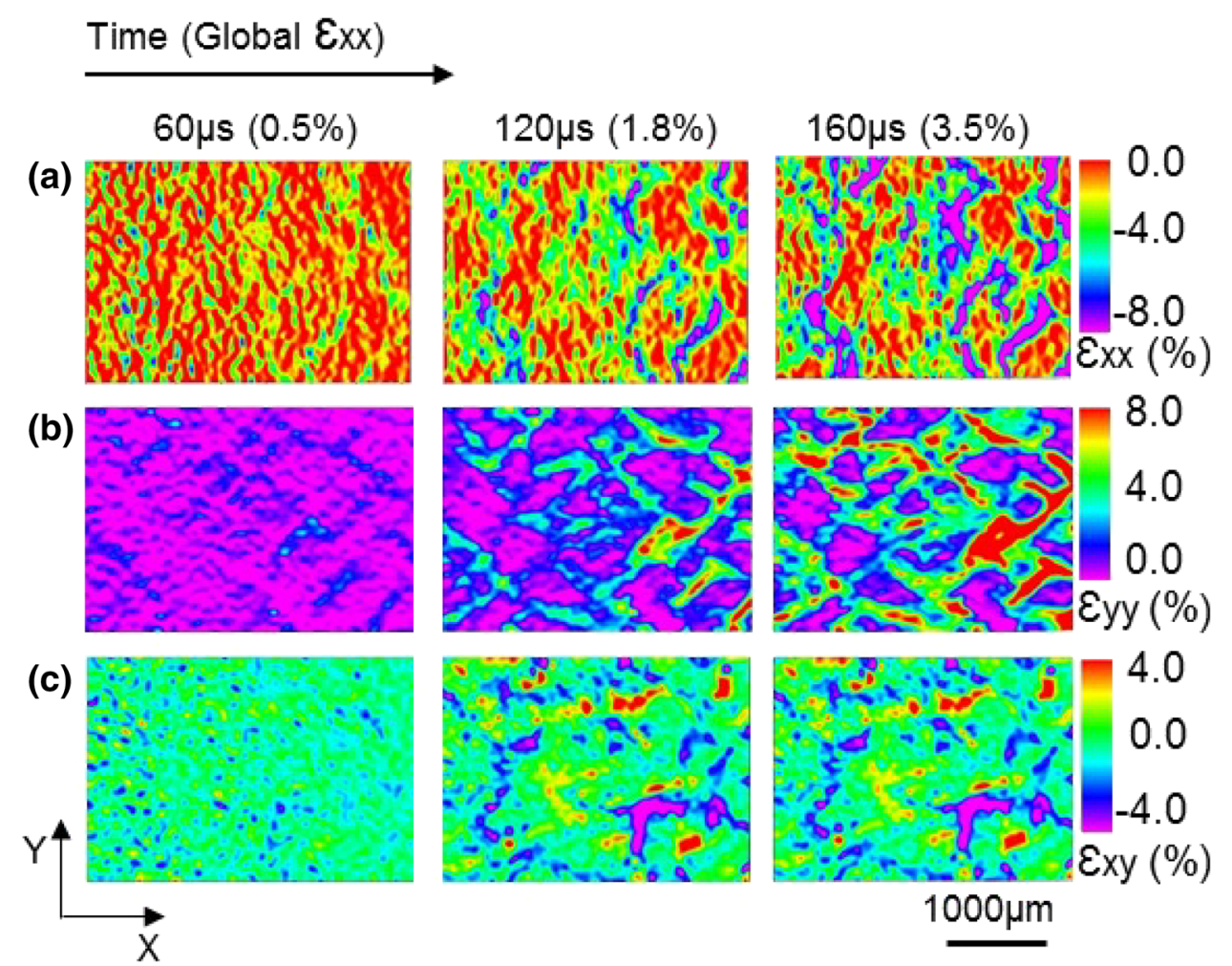

force chains in the material, and there might be some crystals that form favorable paths for the load transfer mechanism. The speculated force chains are based on the continuity of the compressive strain across the specimen length, and are in crystals either in contact or very close to each other, which agrees well with the fact that as the solid 
Fig. 6 a Microstructure of the specimen with crystals labeled and the associated local, b axial, c lateral, and $\mathbf{d}$ shear strain fields (a)

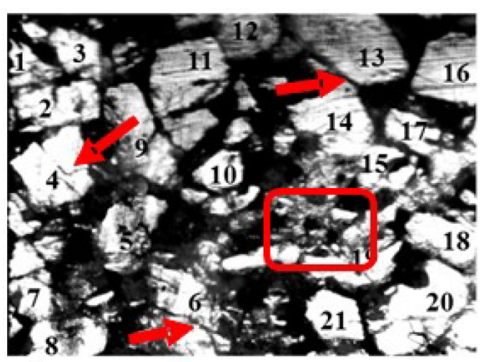

(c)

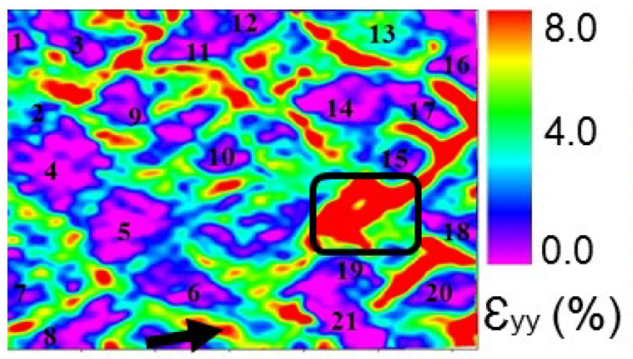

(b)

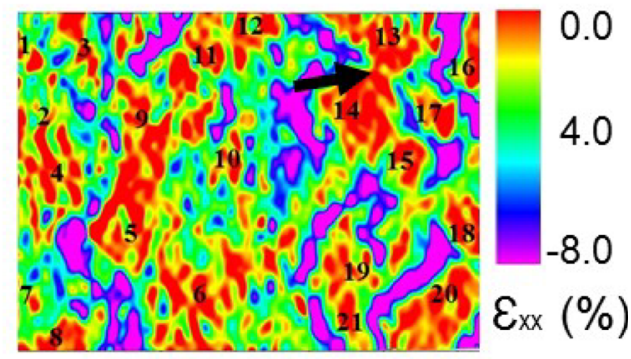

(d)

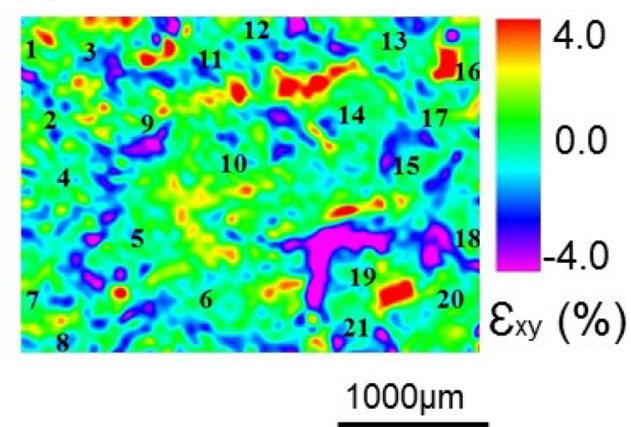

loading increases it results in permanent stress bridging [28]. However considering the percentage of solid loading used in the specimen, additional work is needed at different solid loadings to reach a conclusive observation on the force chain formation in the material.

It is important to note that the lateral strain localization occurred at grain boundaries that are at an angle about $\pm 45^{\circ}$ with respect to the loading direction. Very high tensile strain, up to $8 \%$, has been seen at a small global compressive strain of $0.5 \%$. Figure $6 \mathrm{c}$ shows the comparison of the local transverse strain field with microstructure shown in Fig. 6a. It is shown that the strain localization is substantial in the polymer binder rich area (marked in a black rectangle in Fig. 6c. This area is formed by collections of small crystals bound together by the polymer binder and surrounded by large crystals, which potentially form a weak region for tension. It is also visible that the lateral strain around this region extends to the boundary of the specimen, indicating the possibility of either binder delamination arround the crystal or/and crystal cracking, which will be further discussed in the next section. The local shear strain field is also significantly localized at the boundaries and prevalent at the weak polymer-rich region. The high shear localization could cause the sliding of the crystal over another and crystal rotation.

\section{Crystal Fracture}

One of the major failure mechanisms of PBS observed in this experiment is crystal fracture. As shown in Fig. 6c, a large strain value is clear on or across some crystals, such as $\mathrm{C} 2, \mathrm{C} 9, \mathrm{C} 6$, and $\mathrm{C} 13$. The strain on this crystal is as high as $4 \%$. However, the sugar crystal used in this experiment is brittle and will not be able to deform to a strain of order 3-4\% without fracture. This indicates that some of the crystals undergo fracture. Crystals $\mathrm{C} 9$ and $\mathrm{C} 13$ are relatively large in size, which is a favorable condition for crystal fracture if it is surrounded by large crystals. In such cases, the number of crystal to crystal contact points will be less, which essentially reduces the chances of force distribution through more contact points and hence increases the probability of stress concentration. It is distinctly visible from the Fig. 6a that $\mathrm{C} 14$ and $\mathrm{C} 13$ have a single point contact, where a sharp corner of $\mathrm{C} 14$ hitting C13. Consequently, these conditions cause stress concentration and finally fracturing of $\mathrm{C} 13$. Similar observations can be made on $\mathrm{C} 2$ and $\mathrm{C} 9$ as well. Whereas, in the case of $\mathrm{C} 6$, an initial crack formed from the preparation of the material is visible, see Fig. 6a (red arrow on C6). Fracture of crystals during material preparation is common [29]. This initial crack starts to grow as loading progress and leads to fracture C6. It is very interesting to note that $\mathrm{C} 4$ also has an initial crack as of C6, as indicated in Fig. 6a by an arrow, but there is no further fracture of the crystal is observed. This could be either the disengagement of $\mathrm{C} 4$ in the load transfer mechanism due to a large region of binder ahead of the crystal, possible force chain mechanism or rigid body rotation. Nevertheless, it indicates that increasing the polymer binder will effectively reduce the probability of crystal fracture due to crystal-crystal contact. 
Fig. 7 Angle or rotation of crystals with time a From crystal number 1-10, b from crystal 11-21, c the corresponding microstructure and the rotation direction
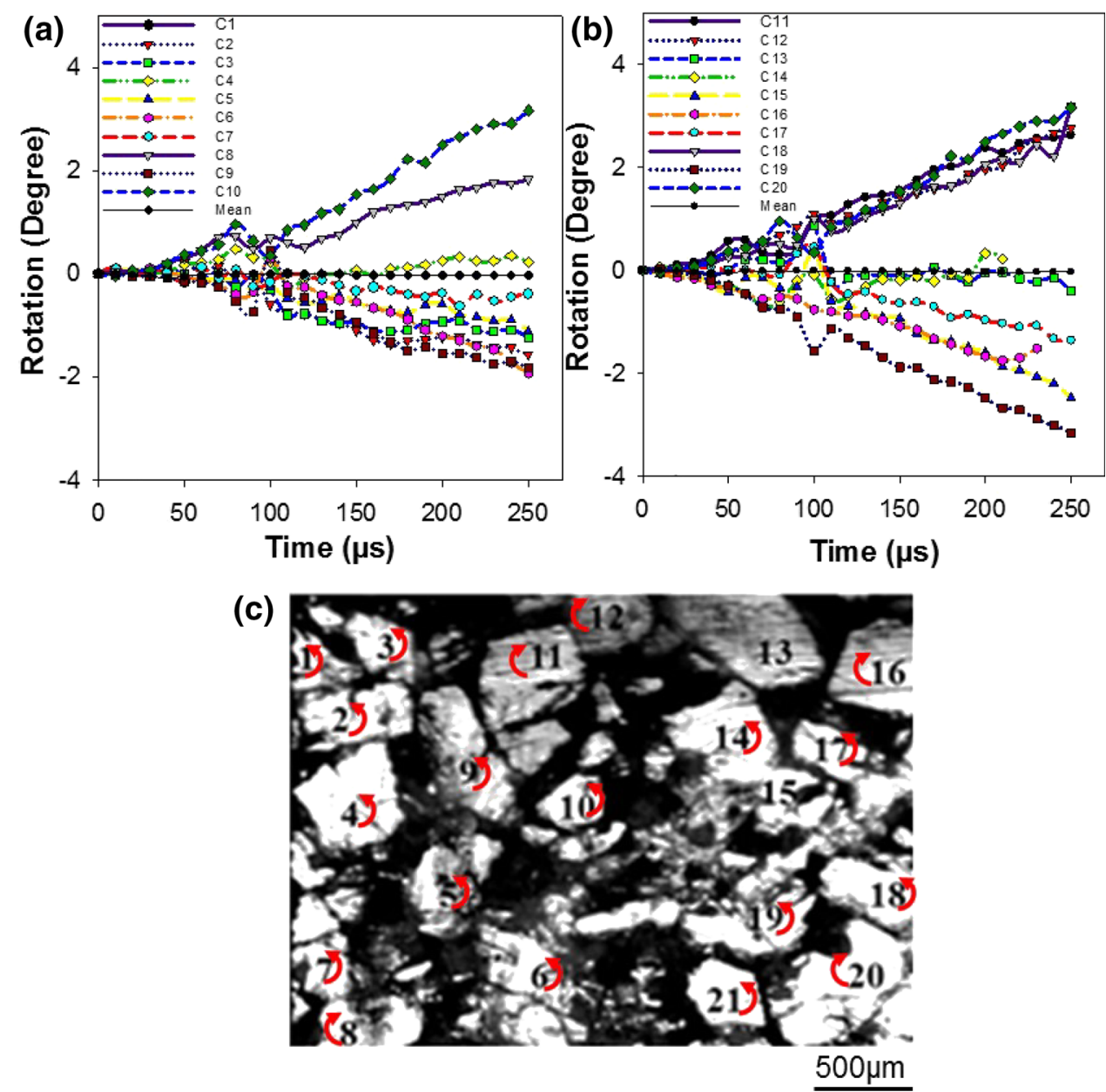

Table 2 Magnitude and direction of crystals rotation

\begin{tabular}{lllll}
\hline Crystals & Rotation direction & Max. rotation (degree) & Min. rotation (degree) & Mean rotation (degree) \\
\hline $\mathrm{C} 1, \mathrm{C} 2, \mathrm{C} 3, \mathrm{C} 4, \mathrm{C} 5, \mathrm{C} 6, \mathrm{C} 7, \mathrm{C} 9$, & Clockwise & $\mathrm{C} 21=3^{\circ}, \mathrm{C} 17=2.5^{\circ}$ & $\mathrm{C} 4, \mathrm{C} 14$ and C16 $=0.2^{\circ}$ & $0.03^{\circ}$ \\
$\mathrm{C} 14, \mathrm{C} 19, \mathrm{C} 18, \mathrm{C} 21$ & & $\mathrm{C} 6=2^{\circ}$ & \\
$\mathrm{C} 8, \mathrm{C} 10, \mathrm{C} 20, \mathrm{C} 11, \mathrm{C} 12$ & Anticlockwise & $\mathrm{C} 10=3.2^{\circ}$, & \\
& & $\mathrm{C} 20=3.2^{\circ}$, & \\
& & $\mathrm{C} 11$ and $\mathrm{C} 12=3.2^{\circ}$ &
\end{tabular}

\section{Crystal Rotation}

Another deformation mechanism observed in this experiment is crystal rotation and sliding. Crystal rotation and sliding have been observed in granular materials under quasi-static and dynamic loading conditions in the past [30, 31]. Figure 7 shows the rotation of each crystal as a function of loading time. The magnitude and direction of rotation varies from grain to grain, and crystal rotation as high as $3.2^{\circ}$ is measured (Table 2). It is also observed that, in some cases, two or more grains form a group and rotate together up to a certain loading time. For example $\mathrm{C} 12$ and C11 form a pair and rotate together up to a time of $200 \mu \mathrm{s}$ in a clockwise direction. There is no relative rotation between $\mathrm{C} 11$ and $\mathrm{C} 12$ until this time. $\mathrm{C} 1, \mathrm{C} 2$, and $\mathrm{C} 3$ have also formed groups and rotate in the same direction. On the other hand, some crystals in contact with another rotating crystal are shown to be stationary or rotates in a different direction, which could create sliding and friction between the adjacent crystals. For example $\mathrm{C} 4$, which is in contact with $\mathrm{C} 2$, does not rotate significantly, which could cause potential sliding friction with $\mathrm{C} 2$. In addition, relative 
rotation between the adjacent crystals can cause delamination at the interface and could be a potential failure mode in PBX.

\section{Conclusions}

An experimental method has been developed to investigate the local deformation mechanism in PBX under dynamic loading conditions by the employed high-speed camera along with high magnification extension tube. To the knowledge of the authors, for the first time, quantitative data at a spatial resolution of $10 \mu \mathrm{m} / \mathrm{pixel}$ and at a framing rate of 100,000 frames/second is achieved. Based on the macro and meso scale experiments the following points are summarized.

1. Macro scale experiments showed that under dynamic loading conditions the PBS fracture by shear band formation and the shear band formation started around $6 \%$ of global axial strain.

2. From the mesoscale experiments different deformation mechanisms are observed.

a. The strain is highly localized in the interface region with thick polymer binder, while the crystals either rotate or realign themselves to accommodate deformation in the binder.

b. Crystals with direct multi-point contact with their neighbor crystal are unlikely to undergo large deformation.

c. On the other hand crystals either with initial crack or in direct point contact with the neighbor crystal are seen to undergo fracture.

d. The lateral strain is highly visible in areas where small crashed crystals are bonded together, indicating a potential spot for opening-mode fracture initiation.

Acknowledgments The financial support of Air Force Office of Scientific Research (AFOSR) under Grant No. FA9550-14-1-0209 is gratefully acknowledged.

\section{References}

1. Roessig KM (2002) Mesoscale mechanics of plastic bonded explosives. In: AIP Conference Proceedings IOP Institute of Physics Publishing Ltd, pp 973-978

2. Peterson PD, Mortensen KS, Idar DJ et al (2001) Strain field formation in plastic bonded explosives under compressional punch loading. J Mater Sci 36:1395-1400

3. Zhou Z, Chen P, Duan Z, Huang F (2012) Study on fracture behaviour of a polymer-bonded explosive simulant subjected to uniaxial compression using digital image correlation method. Strain 48:326-332
4. Prentice HJ, Proud WG, Walley SM, Field JE (2010) The use of digital speckle radiography to study the ballistic deformation of a polymer bonded sugar (an explosive simulant). Int J Impact Eng 37:1113-1120

5. Siviour CR, Grantham SG, Williamson DM et al (2009) Novel measurements of material properties at high rates of strain using speckle metrology. Imaging Sci J 57:326-332

6. Siviour CR, Grantham SG (2009) High resolution optical measurements of specimen deformation in the split Hopkinson pressure bar. Imaging Sci J 57:333-343

7. Grantham SG, Siviour CR, Proud WG, Field JE (2004) Highstrain rate Brazilian testing of an explosive simulant using speckle metrology. Meas Sci Technol 15:1867

8. Siviour C, Laity P, Proud W et al (2008) High strain rate properties of a polymer-bonded sugar: their dependence on applied and internal constraints. Proc R Soc A Math Phys Eng Sci 464:1229-1255. doi:10.1098/rspa.2007.0214

9. Balzer JE, Siviour CR, Walley SM et al (2004) Behaviour of ammonium perchlorate-based propellants and a polymer-bonded explosive under impact loading. Proc R Soc A Math Phys Eng Sci 460:781-806. doi:10.1098/rspa.2003.1188

10. Drodge DR, Williamson DM (2016) Understanding damage in polymer-bonded explosive composites. J Mater Sci 51:668-679

11. Rae PJ, Palmer SJP, Goldrein HT et al (2002) Quasi-static studies of the deformation and failure of PBX 9501. Proc R Soc A Math Phys Eng Sci 458:2227-2242. doi:10.1098/rspa.2002.0967

12. Ravindran S, Kidane A (2016) Meso-Scale Deformation behavior of polymer bonded energetic material under quasi-static compression. In: Mech. Compos. Multi-functional mater, vol 7. Springer, Berlin, pp 345-350

13. Koohbor B, Ravindran S, Kidane A (2015) Meso-scale strain localization and failure response of an orthotropic woven glassfiber reinforced composite. Compos Part B Eng 78:308-318

14. Ravindran S, Koohbor B, Kidane A (2015) On the meso-macro scale deformation of low carbon steel. In: Adv. Opt. Methods Exp. Mech, vol 3. Springer, Berlin, pp 409-414

15. Sutton MA, Li N, Joy DC et al (2007) Scanning electron microscopy for quantitative small and large deformation measurements part I: SEM imaging at magnifications from 200 to 10,000. Exp Mech 47:775-787

16. Kammers AD, Daly S (2013) Digital image correlation under scanning electron microscopy: methodology and validation. Exp Mech 53:1743-1761

17. Zhou Z, Chen P, Huang F, Liu S (2011) Experimental study on the micromechanical behavior of a PBX simulant using SEM and digital image correlation method. Opt Lasers Eng 49:366-370

18. Ravindran S, Tessema A, Kidane A (2015) Note: dynamic mesoscale full field surface deformation measurement of heterogeneous materials. Rev Sci Instrum. Under review

19. Ravindran S, Tessema A, Kidane A, Sutton MA (2016) Mesoscale deformation mechanisms of polymer bonded energetic materials under dynamic loading. In: Mech. Compos. Multifunctional Mater, vol 7. Springer, Berlin, pp 451-456

20. Liu C (2005) On the minimum size of representative volume element: an experimental investigation. Exp Mech 45:238-243

21. Asadi M (2006) Beet-sugar handbook. Wiley, Hoboken

22. Gray III GT, Blumenthal WR, Idar DJ, Cady CM (1998) Influence of temperature on the high-strain-rate mechanical behavior of PBX 9501. In: Tenth Am. Phys. Soc. Top. Conf. shock compression condens. matter. AIP Publishing, pp 583-586

23. Zhao H, Gary G, Klepaczko JR (1997) On the use of a viscoelastic split hopkinson pressure bar. Int $\mathrm{J}$ Impact Eng 19:319-330. doi:10.1016/S0734-743X(96)00038-3

24. Sutton MA, Orteu JJ, Schreier H (2009) Image correlation for shape, motion and deformation measurements: basic concepts, 
theory and applications. Springer Science \& Business Media, New York

25. Mallon S, Koohbor B, Kidane A, Sutton MA (2014) Fracture behavior of prestressed composites subjected to shock loading: a DIC-based study. Exp Mech 55:211-225

26. Koohbor B, Mallon S, Kidane A, Sutton MA (2014) A DIC-based study of in-plane mechanical response and fracture of orthotropic carbon fiber reinforced composite. Compos Part B Eng 66:388-399

27. Herbold EB, Nesterenko VF, Benson DJ et al (2008) Particle size effect on strength, failure, and shock behavior in polytetrafluoroethylene-Al-W granular composite materials. J Appl Phys 104:103903

28. Barua A, Horie Y, Zhou M (2012) Microstructural level response of HMX-Estane polymer-bonded explosive under effects of transient stress waves. In: Proceedings of the Royal Society London A Mathematical, Physical and Engineering Science. The Royal Society, pp 3725-3744

29. Hu Z, Luo H, Bardenhagen $\mathrm{S}$ et al (2015) Internal deformation measurement of polymer bonded sugar in compression by digital volume correlation of tomography. Exp Mech 1:289-300

30. Tordesillas A, Muthuswamy M, Walsh SD (2008) Mesoscale measures of nonaffine deformation in dense granular assemblies. J Eng Mech 134:1095-1113

31. Kuhn MR, Bagi K (2004) Contact rolling and deformation in granular media. Int J Solids Struct 41:5793-5820 